\title{
TOTALLY MONOTONE FUNCTIONS WITH APPLICATIONS TO THE BERGMAN SPACE
}

\author{
B. KORENBLUM, R. O'NEIL, K. RICHARDS, AND K. ZHU
}

\begin{abstract}
Using a theorem of S. Bernstein [1] we prove a special case of the following maximum principle for the Bergman space conjectured by B. Korenblum [3]: There exists a number $\delta \in(0,1)$ such that if $f$ and $g$ are analytic functions on the open unit disk D with $|f(z)| \leq|g(z)|$ on $\delta \leq|z|<1$ then $\|f\|_{2} \leq\|g\|_{2}$, where \|\|$_{2}$ is the $L^{2}$ norm with respect to area measure on D. We prove the above conjecture when either $f$ or $g$ is a monomial; in this case we show that the optimal constant $\delta$ is greater than or equal to $1 / \sqrt{3}$.
\end{abstract}

\section{INTRODUCTION}

A function $f$ on $[0,+\infty)$ is totally monotone if $(-1)^{n} f^{(n)}(x) \geq 0$ for all nonnegative integers $n$ and all $x$ in $[0,+\infty)$. It is clear that if $t$ is nonnegative then the function $f(x)=e^{-t x}$ is totally monotone. It turns out that the functions $e^{-t x}$ are the building blocks for the set of totally monotone functions. S. N. Bernstein [1] proved that a function on $[0,+\infty)$ is totally monotone if and only if there exists a finite positive Borel measure $\sigma$ on $[0,+\infty)$ such that

$$
f(x)=\int_{0}^{\infty} e^{-t x} d \sigma(t), \quad x \in[0,+\infty) .
$$

In this paper we use Bernstein's characterization of totally monotone functions to prove two estimates about the integral of totally monotone functions. Specifically we prove the following theorems.

Theorem A. Suppose $T>0$ and $f(x)$ is totally monotone on $[0,+\infty)$. If $f(x) \geq e^{-T x}$ at the point $x=\frac{1}{T+1}$, then

$$
\int_{0}^{\infty} f(x) d \lambda(x) \geq \int_{0}^{\infty} e^{-T x} d \lambda(x),
$$

where $d \lambda(x)=e^{-x} d x$.

Theorem B. Suppose $T>0$ and $f(x)$ is totally monotone on $[0,+\infty)$. If $f(x) \leq e^{-T x}$ for $0 \leq x \leq 2$, then

$$
\int_{0}^{\infty} f(x) d \lambda(x) \leq \int_{0}^{\infty} e^{-T x} d \lambda(x) .
$$

Received by the editors February 28, 1991.

1980 Mathematics Subject Classification (1985 Revision). Primary 30C80, 26D15; Secondary 26D48, 30D15.

The fourth author's research partially supported by the National Science Foundation. 
As an application of the above results we shall obtain estimates on the Bergman norm of an analytic function on the open unit disk $\mathbf{D}$ in the complex plane C. Recall that the Bergman space of the unit disk D consists of analytic functions $f(z)$ on $\mathbf{D}$ such that

$$
\|f\|^{2}=\int_{\mathbf{D}}|f(z)|^{2} d A(z)<+\infty,
$$

where $d A$ is the normalized area measure on $\mathbf{D}$. $\|f\|$ above is called the Bergman norm of $f$. The following conjecture was made in [3] concerning the Bergman norm of an analytic function on $\mathbf{D}$.

Conjecture. There exists a constant $\delta \in(0,1)$ such that if $f$ and $g$ are analytic in D with $|f(z)| \leq|g(z)|$ for $\delta \leq|z|<1$ then

$$
\int_{\mathbf{D}}|f(z)|^{2} d A(z) \leq \int_{\mathbf{D}}|g(z)|^{2} d A(z) .
$$

Note that the above inequality is trivial if the zeros of $g$ (counting multiplicity) are also zeros of $f$, because the maximum principle then implies that $|f(z)| \leq|g(z)|$ for all $z \in \mathbf{D}$. It was also proved in [3] that the above conjecture is true if the zeros of $f$ (counting multiplicity) are zeros of $g$. In this case the constant $\delta$ is greater than or equal to $1 /\left(2 e^{2}\right)$.

As a consequence of our integral estimates on totally monotone functions we show that the above conjecture holds if one of the two functions $f$ and $g$ is a monomial. In this case we also show that the constant $\delta$ is greater than or equal to $1 / \sqrt{3}$. This answers a question of L. Carleson [2] who originally asked the question for $g(z)=z$.

Theorem C. Suppose $n \geq 0$ is an integer and $f$ is analytic in D. If $|f(z)| \geq\left|z^{n}\right|$ for $1 / \sqrt{2} \leq|z|<1$, then

$$
\int_{\mathbf{D}}|f(z)|^{2} d A(z) \geq \int_{\mathbf{D}}\left|z^{n}\right|^{2} d A(z) .
$$

Theorem D. Suppose $n \geq 0$ is an integer and $f$ is analytic in D. If $|f(z)| \leq$ $\left|z^{n}\right|$ for $1 / \sqrt{3} \leq|z|<1$, then

$$
\int_{\mathbf{D}}|f(z)|^{2} d A(z) \leq \int_{\mathbf{D}}\left|z^{n}\right|^{2} d A(z) .
$$

The rest of the paper is organized as follows. In the next section we shall present a simple proof for Bernstein's characterization of totally monotone functions. In $\S 3$ we prove the lower estimates, Theorems A and C. Section 4 is devoted to the proof of the upper estimates, Theorems B and D. Finally in $\S 5$ we give a counterexample to show that the approach in this paper cannot be used to prove the full conjecture stated earlier.

\section{ANOTHER PROOF OF BERNSTEIN'S THEOREM}

Recall that a function $f$ on $[0,+\infty)$ is totally monotone if $(-1)^{n} f^{(n)}(x) \geq 0$ for all $x \in[0,+\infty)$ and all integers $n \geq 0$. S. N. Bernstein [1] proved that the class of totally monotone functions on $[0,+\infty)$ coincides with the class of functions $f$ with a representation

$$
f(x)=\int_{0}^{\infty} e^{-t x} d \sigma(t), \quad x \in[0,+\infty) .
$$


Bernstein's original proof was based on some deep properties of exponential polynomials. A much simpler proof can be found [4]. However, the publication [4] is not available in English translation and is therefore almost unknown in the West. For the reader's convenience we include the short proof of Bernstein's theorem from [4].

Theorem 1. A function $f$ on $[0,+\infty)$ is totally monotone if and only if there exists a finite positive Borel measure $\sigma$ on $[0,+\infty)$ such that

$$
f(x)=\int_{0}^{\infty} e^{-t x} d \sigma(t), \quad x \in[0,+\infty) .
$$

Proof. A totally monotone function $f$ has the following properties:

(a) $f^{(n)}(x)=o\left(x^{-n}\right) \quad(x \rightarrow+\infty), n \geq 1$;

(b) $\frac{1}{n !} \int_{0}^{\infty} x^{n}\left|f^{(n+1)}(x)\right| d x=f(0)-f(+\infty), n \geq 0$.

In fact, since $(-1)^{n} f^{(n)}(x)$ is nonnegative and nonincreasing, we have for $x>0$ and $n \geq 1$,

$$
\begin{aligned}
\left|f^{(n)}(x)\right| & =(-1)^{n} f^{(n)}(x) \leq \frac{2}{x} \int_{\frac{x}{2}}^{x}(-1)^{n} f^{(n)}(x) d x \\
& =\frac{2}{x}\left|f^{(n-1)}(x)-f^{(n-1)}\left(\frac{x}{2}\right)\right| .
\end{aligned}
$$

Now (a) follows from induction and (b) can be proved by integration by parts:

$$
\begin{aligned}
\frac{1}{n !} \int_{0}^{\infty} x^{n}\left|f^{(n+1)}(x)\right| d x & =\frac{(-1)^{(n+1)}}{n !} \int_{0}^{\infty} x^{n} f^{(n+1)}(x) d x \\
& =\frac{(-1)^{n}}{(n-1) !} \int_{0}^{\infty} x^{(n 11)} f^{(n)}(x) d x \\
& =\cdots=-\int_{0}^{\infty} f^{\prime}(x) d x=f(0)-f(+\infty)
\end{aligned}
$$

To complete the proof of Bernstein's theorem, we observe that the functions

$$
\varphi_{n}(x)= \begin{cases}\left(1-\frac{x}{n}\right)^{n} & (0 \leq x \leq n), \\ 0 & (n \leq x<+\infty),\end{cases}
$$

converge uniformly on $[0,+\infty)$ to the function $e^{-x}$ as $n \rightarrow+\infty$. Applying integration by parts again, we get

$$
\begin{aligned}
f(x)-f(+\infty) & =-\int_{x}^{\infty} f^{\prime}(t) d t=\int_{x}^{\infty}(t-x) f^{\prime \prime}(t) d t \\
& =\cdots=\frac{(-1)^{(n+1)}}{n !} \int_{x}^{\infty}(t-x)^{n} f^{(n+1)}(t) d t \\
& =\frac{(-1)^{(n+1)} n}{n !} \int_{\frac{x}{n}}^{\infty}\left(1-\frac{x}{n t}\right)^{n}(n t)^{n} f^{(n+1)}(n t) d t \\
& =\int_{0}^{\infty} \varphi_{n}\left(\frac{x}{t}\right) d\left(\frac{(-1)^{(n+1)} n}{n !} \int_{0}^{t}(n t)^{n} f^{(n+1)}(n t) d t\right) \\
& =\int_{0}^{\infty} \varphi_{n}(x t) d \sigma_{n}(t),
\end{aligned}
$$


where

$$
\sigma_{n}(t)=\frac{1}{n !} \int_{\frac{1}{t}}^{\infty}(-1)^{(n+1)} n(n t)^{n} f^{(n+1)}(n t) d t .
$$

From property (b) we see that the total variation of $\sigma_{n}$ over $[0,+\infty)$ is

$$
\frac{1}{n !} \int_{0}^{\infty} n(n t)^{n}\left|f^{(n+1)}(n t)\right| d t=f(0)-f(+\infty) \text {. }
$$

By Helly's selection theorem there exists a subsequence $\left\{\sigma_{n_{k}}(t)\right\}$ that converges almost everywhere to a bounded nonnegative nondecreasing function $\sigma(t)$ on $[0,+\infty)$. Finally the uniform convergence of $\varphi_{n}(t)$ allows us to take the limit under the integral sign and obtain

$$
f(x)-f(+\infty)=\int_{0}^{\infty} e^{-t x} d \sigma(t)
$$

or

$$
f(x)=f(+\infty)+\int_{0}^{\infty} e^{-t x} d \sigma(t)
$$

for all $x$ in $[0,+\infty)$. Adding to $\sigma$ a mass $f(+\infty)$ at 0 we obtain Bernstein's theorem.

Suppose $f$ is totally monotone on $[0,+\infty)$ and consider the function

$$
F(x)=f(-\log x), \quad 0 \leq x \leq 1 .
$$

By Bernstein's theorem there exists a positive Borel measure $\sigma$ on $[0,+\infty)$ such that

$$
f(x)=\int_{0}^{\infty} e^{-t x} d \sigma(t)
$$

for all $x$ in $[0,+\infty)$. It follows easily that

$$
F(x)=\int_{0}^{\infty} x^{t} d \sigma(t)
$$

for all $x$ in $[0,1]$. This motivates the following:

Definition. A function $f$ on $[0,1]$ is totally monotone if there exists a positive Borel measure $\sigma$ on $[0,+\infty)$ such that

$$
f(x)=\int_{0}^{\infty} x^{t} d \sigma(t)
$$

for all $x$ in $[0,1]$.

It is clear from the definition that any function $f(x)=x^{t}$ with $t \geq 0$ is totally monotone on $[0,1]$. Similarly, if $a_{k} \geq 0$ for all $k \geq 0$ and

$$
f(x)=\sum_{k=0}^{\infty} a_{k} x^{k}, \quad x \in[0,1],
$$

then $f$ is totally monotone on $[0,1]$. These are the functions that we shall use in the estimates of the Bergman norm of an analytic function on the open unit disk D.

Note that if $f(x)=\sum_{k=0}^{\infty} a_{k} x^{k}$ with $a_{k} \geq 0$ then $f^{(n)}(x) \geq 0$ for all $n \geq 0$ and $x \in[0,1)$. However, it is not true that totally monotone functions on $[0,1]$ always satisfy $f^{(n)}(x) \geq 0$ for all $n \geq 0$ and $x \in[0,1)$. In fact, 
$\sqrt{x}$ is totally monotone on $[0,1]$ according to our definition, but the second derivative of $\sqrt{x}$ is negative on $[0,1)$.

\section{THE LOWER ESTIMATES}

If $f(x) \geq e^{-T x}$ for all $x \in[0,+\infty)$, it is obvious that

$$
\int_{0}^{\infty} f(x) d \lambda(x) \geq \int_{0}^{\infty} e^{-T x} d \lambda(x) .
$$

The main result of this section is that the above integral inequality remains valid if $f$ is totally monotone and $f(x) \geq e^{-T x}$ holds at the single point $x=\frac{1}{T+1}$. The result is sharp in the sense that any other point (different from $\frac{1}{T+1}$ ) will make the above integral estimate false.

Theorem 2. Suppose $T>0$ and $f$ is totally monotone on $[0,+\infty)$. If $f(x) \geq$ $e^{-T x}$ at $x=\frac{1}{T+1}$, then

$$
\int_{0}^{\infty} f(x) d \lambda(x) \geq \int_{0}^{\infty} e^{-T x} d \lambda(x),
$$

where $d \lambda(x)=e^{-x} d x$.

Proof. By Bernstein's theorem, there is a positive Borel measure $\sigma$ on $[0,+\infty)$ such that

$$
f(x)=\int_{0}^{\infty} e^{-t x} d \sigma(t)
$$

for all $x \geq 0$. For any $a \in[0,1]$ we have

$$
\begin{aligned}
\int_{0}^{\infty} f(x) d \lambda(x) & =\int_{0}^{\infty}\left(\int_{0}^{\infty} e^{-t x} d \sigma(t)\right) e^{-x} d x \\
& =\int_{0}^{\infty} \frac{d \sigma(t)}{1+t}=\int_{0}^{\infty} e^{-a t} \cdot \frac{e^{a t}}{1+t} \cdot d \sigma(t) \\
& \geq\left[\int_{0}^{\infty} e^{-a t} d \sigma(t)\right]\left[\inf _{t>0} \frac{e^{a t}}{1+t}\right] \\
& =f(a) a e^{1-a} .
\end{aligned}
$$

Substituting $a=\frac{1}{T+1}$ we obtain

$$
\int_{0}^{\infty} f(x) d \lambda(x) \geq \frac{1}{T+1} e^{\frac{T}{T+1}} f\left(\frac{1}{T+1}\right) \geq \frac{1}{T+1}=\int_{0}^{\infty} e^{-T x} d \lambda(x) .
$$

This completes the proof of Theorem 2 .

Corollary 3. Suppose $\alpha>0$ and $f$ is totally monotone on $[0,1]$. If $f(x) \geq x^{\alpha}$ at $x=\exp \left(-\frac{1}{\alpha+1}\right)$, then

$$
\int_{0}^{1} f(x) d x \geq \int_{0}^{1} x^{\alpha} d x
$$

Proof. Let $x=e^{-t}$. Then

$$
\int_{0}^{1} f(x) d x=\int_{0}^{\infty} f\left(e^{-t}\right) d \lambda(t)
$$


and

$$
\int_{0}^{1} x^{\alpha} d x=\int_{0}^{\infty} e^{-\alpha t} d \lambda(t)
$$

Since $f$ is totally monotone on $[0,1]$, the function $g(t)=f\left(e^{-t}\right)$ is totally monotone on $[0,+\infty)$. Also $f(x) \geq x^{\alpha}$ at $x=\exp \left(-\frac{1}{\alpha+1}\right)$ implies that $g(t) \geq$ $e^{-\alpha t}$ at $t=\frac{1}{\alpha+1}$. The desired result now follows from Theorem 2 .

Corollary 4. Suppose $n \geq 0$ is an integer and $f$ is analytic in D. If $|f(z)| \geq$ $\left|z^{n}\right|$ for $|z|=\exp \left(-\frac{1}{2(n+1)}\right)$, then

$$
\int_{\mathbf{D}}|f(z)|^{2} d A(z) \geq \int_{\mathbf{D}}\left|z^{n}\right|^{2} d A(z)
$$

Proof. Let $f(z)=\sum_{k=0}^{\infty} a_{k} z^{k}$. Then for $x=\exp \left(-\frac{1}{2(n+1)}\right)$ we have

$$
\sum_{k=0}^{\infty}\left|a_{k}\right|^{2} x^{2 k}=\frac{1}{2 \pi} \int_{0}^{2 \pi}\left|f\left(x e^{i t}\right)\right|^{2} d t \geq \frac{1}{2 \pi} \int_{0}^{2 \pi}\left|x^{n} e^{i n t}\right|^{2} d t=x^{2 n}
$$

It follows that

$$
\sum_{k=0}^{\infty}\left|a_{k}\right|^{2} x^{k} \geq x^{n}
$$

for all $x=\exp \left(-\frac{1}{n+1}\right)$. Since the function $\sum_{k=0}^{\infty}\left|a_{k}\right|^{2} x^{k}$ is totally monotone on $[0,1]$, Corollary 3 gives

$$
\int_{0}^{1}\left(\sum_{k=0}^{\infty}\left|a_{k}\right|^{2} x^{k}\right) d x \geq \int_{0}^{1} x^{n} d x .
$$

This is exactly the desired inequality

$$
\int_{\mathbf{D}}|f(z)|^{2} d A(z) \geq \int_{\mathbf{D}}\left|z^{n}\right|^{2} d A(z)
$$

Remark. The constant $\exp \left(-\frac{1}{2(n+1)}\right)$ in the above corollary is not unique (at least in the cases $n=0$ and $n=1)$. In fact, for $n=0$ and $n=1$ we can replace the constant by $1 / \sqrt{2}$. Let $F(x)$ denote the function $\sum_{k=0}^{\infty}\left|a_{k}\right|^{2} x^{k}$, then $F(x)$ is convex in $[0,1]$ and hence

$$
F(x) \geq F\left(\frac{1}{2}\right)+F^{\prime}\left(\frac{1}{2}\right)\left(x-\frac{1}{2}\right)
$$

for all $x$ in $[0,1]$. If $F(x) \geq x^{n}$ at $x=\frac{1}{2}$, then

$$
\int_{0}^{1} F(x) d x \geq \int_{0}^{1}\left[F\left(\frac{1}{2}\right)+F^{\prime}\left(\frac{1}{2}\right)\left(x-\frac{1}{2}\right)\right] d x=F\left(\frac{1}{2}\right) \geq\left(x^{n}\right)\left(\frac{1}{2}\right) .
$$

In the case $n=0,1$, we clearly have $\left(x^{n}\right)\left(\frac{1}{2}\right)=\int_{0}^{1} x^{n} d x$.

Corollary 5. Suppose $n \geq 0$ is an integer and $f$ is analytic in D. If $|f(z)| \geq$ $\left|z^{n}\right|$ for $1 / \sqrt{2} \leq|z|<1$, then

$$
\int_{\mathbf{D}}|f(z)|^{2} d A(z) \geq \int_{\mathbf{D}}\left|z^{n}\right|^{2} d A(z)
$$

Proof. This follows from Corollary 4 and the above remark.

\section{THE UPPER ESTIMATES}

This section is devoted to the proof of Theorems B and D stated in the introduction. 
Theorem 6. Suppose $T>0$ and $f$ is totally monotone on $[0,+\infty)$. If $f(x) \leq$ $e^{-T x}$ for $0 \leq x \leq 2$, then

$$
\int_{0}^{\infty} f(x) d \lambda(x) \leq \int_{0}^{\infty} e^{-T x} d \lambda(x)
$$

where $d \lambda(x)=e^{-x} d x$.

Proof. We shall only need the assumption that $f(x) \leq e^{-T x}$ for $x=0$ and $x=2$. By Theorem 1 we can find a positive Borel measure $\sigma$ on $(0,+\infty)$ such that

$$
f(x)=\int_{0}^{\infty} e^{-t x} d \sigma(t), \quad x \in[0,+\infty) .
$$

Applying Fubini's theorem we easily obtain

$$
\int_{0}^{\infty} f(x) d \lambda(x)=\int_{0}^{\infty} \frac{d \sigma(t)}{t+1} .
$$

Consider the function

$$
g(x)=A+B e^{-2 x}, \quad x \geq 0,
$$

where $A$ and $B$ are determined by the conditions

$$
g(T)=\frac{1}{T+1}, \quad g^{\prime}(T)=-\frac{1}{(T+1)^{2}} .
$$

It is easy to see that

$$
A=\frac{1}{T+1}-\frac{1}{2(T+1)^{2}}, \quad B=\frac{e^{2 T}}{2(T+1)^{2}} .
$$

Let

$$
h(x)=\frac{1}{x+1}-g(x), \quad x \geq 0
$$

Then

$$
\begin{aligned}
h^{\prime}(x) & =-\frac{1}{(x+1)^{2}}+2 B e^{-2 x} \\
& =-\frac{1}{(x+1)^{2}}+\frac{e^{2 T} \cdot e^{-2 x}}{(T+1)^{2}} \\
& =e^{-2 x}\left[\frac{e^{2 T}}{(T+1)^{2}}-\frac{e^{2 x}}{(x+1)^{2}}\right] .
\end{aligned}
$$

It is easy to show that the function $e^{2 x} /(x+1)^{2}$ is increasing on $[0,+\infty)$. Thus $h^{\prime}(x) \leq 0$ for $x \geq T$ and $h^{\prime}(x) \geq 0$ for $0 \leq x \leq T$. This implies that $h(x)$ attains its maximum value at $x=T$ and we must have $h(x) \leq h(T)=0$ for all $x \geq 0$. In other words,

$$
\frac{1}{x+1} \leq A+B e^{-2 x}
$$


for all $x \geq 0$. It follows that

$$
\begin{aligned}
\int_{0}^{\infty} f(x) d \lambda(x) & =\int_{0}^{\infty} \frac{d \sigma(x)}{x+1} \\
& \leq A \int_{0}^{\infty} d \sigma(x)+B \int_{0}^{\infty} e^{-2 x} d \sigma(x) \\
& =A f(0)+B f(2) \leq A+B e^{-2 T} \\
& =\frac{1}{T+1}=\int_{0}^{\infty} e^{-T x} d \lambda(x),
\end{aligned}
$$

completing the proof of Theorem 6 .

Corollary 7. Suppose $\alpha>0$ and $f$ is totally monotone on [0,1]. If $f(x) \leq x^{\alpha}$ for $1 / e^{2} \leq x<1$, then

$$
\int_{0}^{1} f(x) d x \leq \int_{0}^{1} x^{\alpha} d x .
$$

Proof. This follows from Theorem 6 and the change of variables $x=e^{-t}$. See the proof of Corollary 3.

Corollary 8. Suppose $n \geq 0$ is an integer and $f$ is analytic in D. If $|f(z)| \leq$ $\left|z^{n}\right|$ for $1 / e \leq|z| \leq 1$, then

$$
\int_{\mathbf{D}}|f(z)|^{2} d A(z) \leq \int_{\mathbf{D}}\left|z^{n}\right|^{2} d A(z) .
$$

Proof. Let $f(z)=\sum_{k=0}^{\infty} a_{k} z^{k}$. Then $|f(z)| \leq\left|z^{n}\right|$ for $1 / e \leq|z| \leq 1$ implies that

$$
\sum_{k=0}^{\infty}\left|a_{k}\right|^{2} x^{2 k}=\frac{1}{2 \pi} \int_{0}^{2 \pi}\left|f\left(x e^{i t}\right)\right|^{2} d t \leq \frac{1}{2 \pi} \int_{0}^{2 \pi}\left|x^{n} e^{i n t}\right|^{2} d t=x^{2 n}
$$

for all $1 / e \leq x \leq 1$. It follows that

$$
\sum_{k=0}^{\infty}\left|a_{k}\right|^{2} x^{k} \leq x^{n}
$$

for all $1 / e^{2} \leq x \leq 1$ and hence

$$
\int_{0}^{1}\left[\sum_{k=0}^{\infty}\left|a_{k}\right|^{2} x^{k}\right] d x \leq \int_{0}^{1} x^{n} d x
$$

since the function $\sum_{k=0}^{\infty}\left|a_{k}\right|^{2} x^{k}$ is totally monotone on $[0,1]$. This finishes the proof of Corollary 8 .

Remark. The constant $1 / e$ in Corollary 8 above is not best possible. The following theorem improves the constant to $1 / \sqrt{3}$.

Theorem 9. Suppose $n \geq 0$ is an integer and $f$ is an analytic function in $\mathbf{D}$. If $|f(z)| \leq\left|z^{n}\right|$ for $1 / \sqrt{3} \leq|z| \leq 1$, then

$$
\int_{\mathbf{D}}|f(z)|^{2} d A(z) \leq \int_{\mathbf{D}}\left|z^{n}\right|^{2} d A(z) .
$$

Proof. It is easy to see that this is a direct consequence of the following result. 
Theorem 10. Suppose $n \geq 0$ is an integer and $F(x)=\sum_{k=0}^{\infty} a_{k} x^{k}$ with nonnegative coefficients. If $F(x) \leq x^{n}$ for $1 / 3 \leq x \leq 1$, then

$$
\int_{0}^{1} F(x) d x \leq \int_{0}^{1} x^{n} d x=\frac{1}{n+1} .
$$

Proof. The case $n=0$ is trivial. We assume $n \geq 1$ in the rest of the proof.

That $F(x) \leq x^{n}$ for $1 / 3 \leq x \leq 1$ implies that either $F(x) \leq x^{n}$ for all $x \in[0,1]$ or there exists $c \in(0,1 / 3]$ such that $F(c)=c^{n}$. In the first case the conclusion is obvious. So we assume that there exists $c \in(0,1 / 3]$ such that $F(c)=c^{n}$ and $F(x) \leq x^{n}$ for $c \leq x \leq 1$. Thus we have

$$
\begin{gathered}
a_{0}+a_{1}+\cdots+a_{n}+a_{n+1}+\cdots=F(1), \\
a_{0}+a_{1} c+\cdots+a_{n} c^{n}+a_{n+1} c^{n+1}+\cdots=c^{n}
\end{gathered}
$$

with $F(1) \leq 1$. We solve for $a_{n}$ and $a_{n+1}$ in terms of the other terms. Multiplying the first equation above by $-c^{n}$ and then adding the result to the second, we get

$$
a_{n+1}\left(c^{n+1}-c^{n}\right)+\sum_{k \neq n, n+1} a_{k}\left(c^{k}-c^{n}\right)=c^{n}(1-F(1)),
$$

or

$$
a_{n+1}=\sum_{k \neq n, n+1} a_{k} \frac{c^{k}-c^{n}}{c^{n}-c^{n+1}}-\frac{1-F(1)}{1-c} .
$$

Similarly, we obtain

$$
a_{n}=-\sum_{k \neq n, n+1} a_{k} \frac{c^{k}-c^{n+1}}{c^{n}-c^{n+1}}+\frac{1-c F(1)}{1-c} .
$$

It follows that

$$
\begin{array}{rl}
\int_{0}^{1} & F(x) d x=\sum_{k=0}^{\infty} \frac{a_{k}}{k+1} \\
= & \sum_{k \neq n, n+1} \frac{a_{k}}{k+1}+\frac{a_{n}}{n+1}+\frac{a_{n+1}}{n+2} \\
= & \sum_{k \neq n, n+1} a_{k}\left[\frac{1}{k+1}-\frac{c^{k}-c^{n+1}}{(n+1)\left(c^{n}-c^{n+1}\right)}+\frac{c^{k}-c^{n}}{(n+2)\left(c^{n}-c^{n+1}\right)}\right] \\
& +\frac{1}{n+1}+\frac{1-F(1)}{1-c}\left(\frac{c}{n+1}-\frac{1}{n+2}\right) .
\end{array}
$$

Since $c \leq 1 / 3$ and $n \geq 1$, we clearly have

$$
\frac{c}{n+1}-\frac{1}{n+2} \leq 0 \text {. }
$$

But $1-F(1) \geq 0$ and $1-c>0$, we obtain

$$
\begin{aligned}
\int_{0}^{1} F(x) d x \leq & \frac{1}{n+1} \\
& \quad+\sum_{k \neq n, n+1} a_{k}\left[\frac{1}{k+1}-\frac{c^{k}-c^{n+1}}{(n+1)\left(c^{n}-c^{n+1}\right)}+\frac{c^{k}-c^{n}}{(n+2)\left(c^{n}-c^{n+1}\right)}\right] .
\end{aligned}
$$


Writing $c^{k}-c^{n}=c^{k}-c^{n+1}-\left(c^{n}-c^{n+1}\right)$ in the above series, we easily get

$$
\begin{aligned}
\int_{0}^{1} F(x) d x \leq & \frac{1}{n+1} \\
& +\sum_{k \neq n, n+1} a_{k}\left[\frac{1}{k+1}-\frac{1}{n+2}-\frac{c^{k}-c^{n+1}}{(n+1)(n+2)\left(c^{n}-c^{n+1}\right)}\right] .
\end{aligned}
$$

The desired result now follows from the following lemma.

Lemma 11. Let

$$
D(k, n)=\frac{1}{k+1}-\frac{1}{n+2}-\frac{c^{k}-c^{n+1}}{(n+1)(n+2)\left(c^{n}-c^{n+1}\right)} .
$$

Then $D(k, n) \leq 0$ for all $n \geq 1, k \geq 0$, and $c \in(0,1 / 3]$.

Proof. First note that $D(k, n)=0$ if $k=n$ or $n+1$. If $k \geq n+2$, we have

$$
\begin{aligned}
D(k, n) & \leq \frac{1}{n+3}-\frac{1}{n+2}+\frac{c^{n+1}}{(n+1)(n+2)\left(c^{n}-c^{n+1}\right)} \\
& =\frac{c}{(n+1)(n+2)(1-c)}-\frac{1}{(n+2)(n+3)} \\
& =\frac{1}{(n+1)(n+3)(1-c)}\left(2 c-\frac{n+1}{n+2}\right) \\
& \leq \frac{1}{(n+1)(n+3)(1-c)}\left(\frac{2}{3}-\frac{n+1}{n+2}\right) \\
& \leq 0
\end{aligned}
$$

It remains to prove the result for $0 \leq k \leq n-1$.

When $k=0$ and $n=1$, a little simplification shows that

$$
D(0,1)=\frac{1}{2 c}\left(c-\frac{1}{3}\right) \leq 0 .
$$

When $k=0$ and $n \geq 2$, we have

$$
\begin{aligned}
D(k, n) & =1-\frac{1}{n+2}-\frac{1+c+\cdots+c^{n}}{(n+1)(n+2) c^{n}} \\
& \leq \frac{n+1}{n+2}-\frac{1}{(n+1)(n+2) c^{n}} \\
& =\frac{n+1}{(n+2)} c^{n}\left(c^{n}-\frac{1}{(n+1)^{2}}\right) \\
& \leq \frac{n+1}{(n+2)} c^{n}\left(\frac{1}{3^{n}}-\frac{1}{(n+1)^{2}}\right) \\
& \leq 0
\end{aligned}
$$

To prove the remaining case $1 \leq k \leq n-1$, we write

$$
D(k, n)=\frac{n-k+1}{(k+1)(n+2)}-\frac{1-c^{n-k+1}}{(n+1)(n+2)(1-c) c^{n-k}} .
$$

Since $n-k+1 \geq 2$, we have

$$
1-c^{n-k+1} \geq 1-c^{2}>1-c .
$$


It follows that

$$
\begin{aligned}
D(k, n) & \leq \frac{n-k+1}{(k+1)(n+2)}-\frac{1}{(n+1)(n+2) c^{n-k}} \\
& =\frac{n-k+1}{c^{n-k}(k+1)(n+2)}\left(c^{n-k}-\frac{k+1}{(n+1)(n-k+1)}\right) .
\end{aligned}
$$

Let $m=n-k$, then $m \geq 1$ and

$$
D(k, n) \leq \frac{m+1}{c^{m}(k+1)(m+k+2)}\left(c^{m}-\frac{k+1}{(m+1)(m+k+1)}\right) .
$$

It is clear that for $k \geq 1$

$$
\frac{k+1}{(m+1)(m+k+1)} \geq \frac{2}{(m+1)(m+2)} .
$$

Thus

$$
\begin{aligned}
D(k, n) & \leq \frac{m+1}{c^{m}(k+1)(m+k+2)}\left(c^{m}-\frac{2}{(m+1)(m+2)}\right) \\
& \leq \frac{m+1}{c^{m}(k+1)(m+k+2)}\left(\frac{1}{3^{m}}-\frac{2}{(m+1)(m+2)}\right) \\
& \leq 0,
\end{aligned}
$$

completing the proof of Lemma 11.

\section{A COUNTEREXAMPLE}

It is very natural to ask whether the conjecture stated in the Introduction can be proved by using the method of this paper. In other words, one asks if the following is true: there exists a constant $\delta \in(0,1)$ such that if $F(x)=$ $\sum_{k=0}^{\infty} a_{k} x^{k}, G(x)=\sum_{k=0}^{\infty} b_{k} x^{k}, a_{k} \geq 0, b_{k} \geq 0(k \geq 0)$, and $F(x) \leq G(x)$ for $\delta \leq x<1$, then

We show that this is false in general.

$$
\int_{0}^{1} F(x) d x \leq \int_{0}^{1} G(x) d x
$$

For any positive integer $n$ let

$$
P(x)=\left(x-\frac{1}{n+1}\right)(1-x)^{n}=\sum_{k=0}^{n+1} p_{k} x^{k} .
$$

It is clear that $P(x) \geq 0$ for $1 /(n+1) \leq x \leq 1$ and it is easy to show that $\int_{0}^{1} P(x) d x<0$. Let $\left\{a_{k}\right\}$ be a sequence of nonnegative numbers such that $a_{k} \geq\left|p_{k}\right|$ for $0 \leq k \leq n+1$. Now let

$$
F(x)=\sum_{k=0}^{\infty} a_{k} x^{k}, \quad G(x)=F(x)+P(x) .
$$

Then all the coefficients of $F$ and $G$ are nonnegative, $G(x) \geq F(x)$ for $1 /(n+1) \leq x<1$, but

$$
\int_{0}^{1} G(x) d x<\int_{0}^{1} F(x) d x .
$$

Since $n$ is arbitrary, no $\delta$ exists with the desired property. 


\section{REFERENCES}

1. S. N. Bernstein, Sur les fonctions absolument monotones, Acta Math. 52 (1929), 1-66.

2. L. Carleson, Private communication with the first-named author.

3. B. Korenblum, A maximum principle for the Bergman space, Publ. Math. 35 (1991), 479486.

4. _ On two theorems from the theory of absolutely monotone functions, Uspekhi Mat. Nauk 4 (44) (1952), 172-175. (Russian)

(B. Korenblum, R. O'Neil, and K. Zhu) Department of Mathematics, State University OF New York, Albany, New York 12222

(K. Richards) Department of Mathematics, Texas Tech University, Lubbock, Texas 79409 